\title{
Closed-loop control of quasi-static MOEMS mirrors
}

Dominik Holzmann, Andreas Tortschanoff, Martin Lenzhofer Carinthian Tech Research AG, Europastraße 4/1, 9524 Villach, Austria 43 (0)4242 56300 261, dominik.holzmann@ctr.at

\begin{abstract}
A discrete closed-loop control system for fast and accuratepositioning of micro-opto-electromechanical (MOEMS) mirrors is presented is this paper, with special focus on electromagnetically driven quasi-static devices. Through simplified modeling, classic linear control methods can be used for the basically non-linear mirrors. A microcontroller based discrete implementation of the control loop gives flexibility in design and adjustment of different controllers. Compared to openloop, the settling time can be reduced by a factor of 30 using the closed-loop controller.
\end{abstract}

Keywords: MEMS, MOEMS mirror, closed loop, control, quasi-static mirror, digital signal processor

\section{INTRODUCTION}

MEMS mirrors attract more and more interest in miniaturized applications like for example compact projection devices, microscanners, or spectrometers. Quasistatic MOEMS mirrors are of special interest for specific applications, since they enable arbitrary scan trajectories. In order to optimize settling times and the accuracy of the mirror motion, closed loop control is desirable because MOEMS elements can show a significant spread in their mechanical properties like resonance frequency and response curve.

Analog PID [1], digital open loop [2] and digital adaptive closed loop control have been implemented to position MOEMS mirrors. Due to the non-linear behavior of the mirrors and the deviation in their mechanical properties, accurate modeling and the control design is not trivial.

In this paper we describe the design and implementation of a robust closed loop control for MOEMS mirrors based on a simplified mirror model. The TALB1000B mirrors from Texas Instruments (shown in Figure 1) were used for this work [3]. This special device is a two axis mirror which allows $+/-5$ degrees of independent mechanical rotation of in each axis. The rotation of the mirror follows an electromagnetic principle, where the deflection is proportional to the driving current. The resonant frequencies of both axes are in the range of $120 \mathrm{~Hz}$.

Further the on-board optical position feedback can be used for a closed loop control. A sophisticated open - loop [2] but no closed

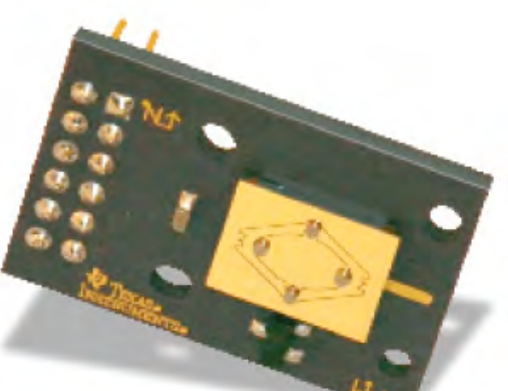

Figure 1: Texas Instruments TALB1000B loop control have been described for this mirror.

\section{SYSTEM MODELING}

From a physical point of view the mirrors behave like a spring-mass system, where the mirror represents the mass and the suspensions the springs. Accurate modeling of quasi-static MOEMS mirrors is quite challenging. In difference to the ideal oscillator, the gain, the damping ratio and the eigenfrequency are not constant but vary with the actual angular deflection and the angular velocity of the mirror. There is also a noticeable variation in these parameters between different mirrors of the same type. Detailed modeling of MOEMS mirrors has been handled in literature and can be found for example in [6] or [7]. 
To simplify the controller design, a constant and linear plant model is desirable. Neglecting the nonlinear parts, the mirrors can be approximately modeled as an underdamped second order system. In this case the transfer function $\mathrm{G}(\mathrm{s})$ can be expressed as follows:

$$
G(s)=\frac{Y(s)}{U(s)}=\frac{\omega_{n}^{2}}{s^{2}+2 \zeta \omega_{n} s+\omega_{n}^{2}}
$$

\begin{tabular}{|l|l|}
\hline Parameter & Value \\
\hline Natural frequency $\omega_{n}$ & $739.46 \frac{\mathrm{rad}}{\mathrm{sec}}$ \\
\hline Damping ratio $\zeta$ & 0.003 \\
\hline
\end{tabular}

Table 1: Mirror parameters

The natural frequency $\omega_{n}$ and the damping ratio $\zeta$ of the TI-mirrors have been determined from an experimental step response and can be found in Table 1. Figure 2shows the simulated and experimental step response for an open - loop input step. The overall behavior of the simplified model and the real mirror is quite similar. Due to simplifications in the model, a perfect match is not expected. For the control design this simplified model is already sufficient.

The TALB1000B mirror is electromagnetically actuated and can be driven basically using a voltage or current driver. In our setup a voltage driver was used. The linear

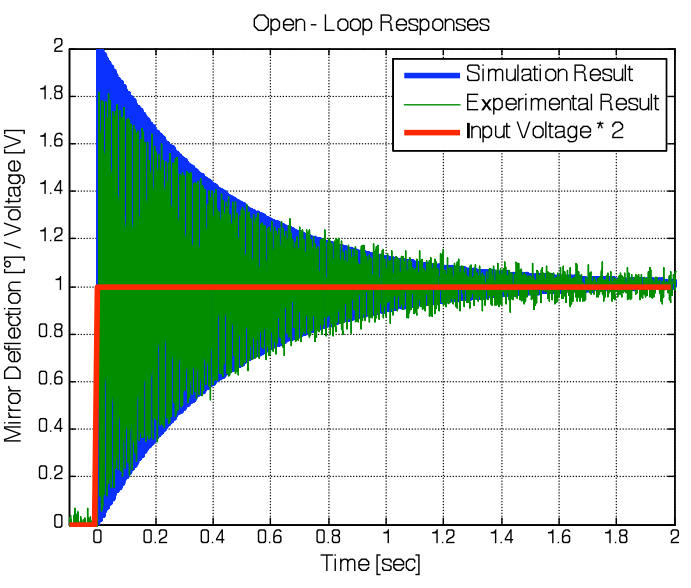

Figure 2: Open loop step responses behavior of the mechanical mirror rotation depending on the drive voltage is shown in Figure $3 \mathrm{a}$. The detection of the actual mirror deflection,which is needed for the closed-loop control,was done using the mirrors internal position feedback.Figure 3bshows the linear behavior of the internal position feedback depending on the mechanical rotation angle.

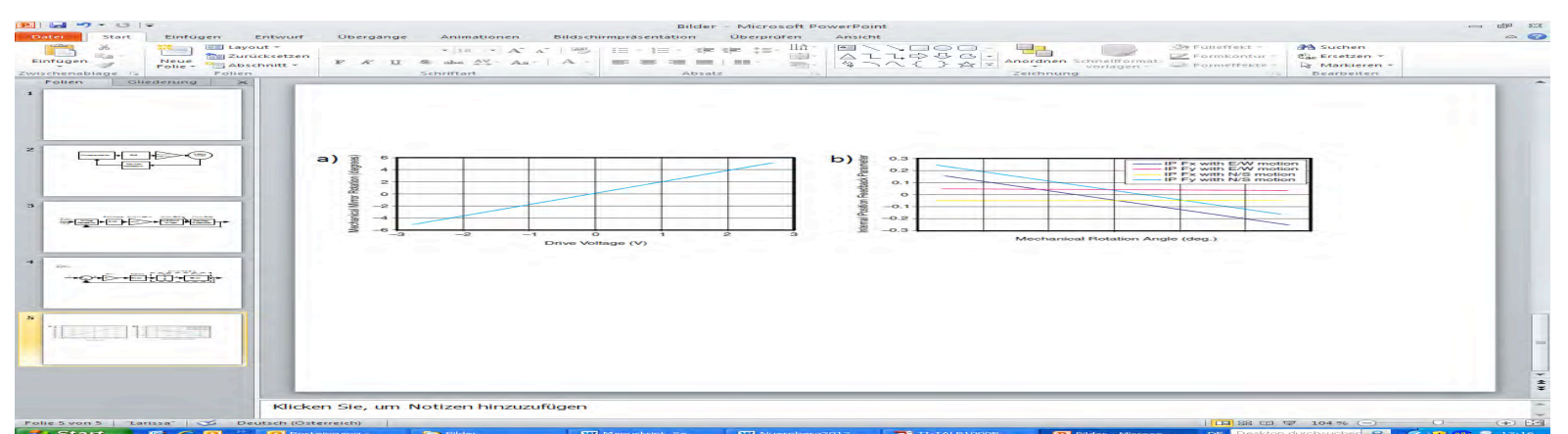

Figure 3: a) Mirror rotation as function of the drive voltage. b) Position feedback as function of the rotation angle. Both taken from [3].

\section{CONTROL DESIGN}

The controller has to guarantee accurate positioning and improve the dynamics of the mirror. Also the natural oscillations of the mirror should be suppressed. Because of the deviations in the mirror parameters a robust control system is required to avoid steady state errors. Further, to realize the control algorithm efficiently even on small scale DSPs or Microcontrollers, it should be as simple as possible. Therefore sophisticated control techniques like adaptive control were avoided. Based on the linearized mirror model, various classic control approaches were implemented. Among them were PI, Lead/Lag with integrator and pole placement controllers. 
Best results were achieved with the pole placement controller. The poles for the closed loop transfer function were chosen following the ITAE (integral of time multiplied by absolute error) optimal designcriteria for step reference inputs [4]. The unity feedback configuration shown in Figure 4was chosen for the control loop. It contains anadditional gain $\mathrm{k}$, the controller $\mathrm{C}(\mathrm{s})$ and the artificial plant $\mathrm{G}_{\text {art }}(\mathrm{s})$. By combining the real plant $\mathrm{G}(\mathrm{s})$ and the integrator to the artificial plant $\mathrm{G}_{\text {art }}(\mathrm{s})$, a robust control system with zero position error is guaranteed independently from the calculated controller $\mathrm{C}(\mathrm{s})$.

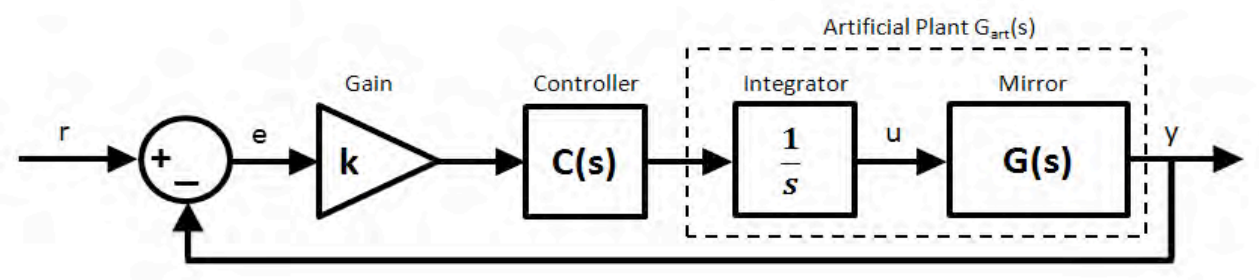

Figure 4: Unity feedback system

Since the artificial plant is of third order, the controller should be of second order and the overall transfer function $T(s)$ of fifth order. With the artificial plant $G_{\text {art }}(s)$ and the denominator for the overall transfer function given, the controller $\mathrm{C}(\mathrm{s})$ can be easily calculated by solving the Diophantine equation. For a fifth order transfer function the denominator is

$$
s^{5}+2.8 \omega_{0} s^{4}+5 \omega_{0}^{2} s^{3}+5.5 \omega_{0}^{3} s^{2}+3.4 \omega_{0}{ }^{4} s+\omega_{0}{ }^{5} .
$$

Expressing the transfer functions in terms of numerator and denominator we get

$$
C(s)=\frac{N_{C}(s)}{D_{C}(s)}, \quad G_{\text {art }}(s)=\frac{N_{G_{a r t}}(s)}{D_{G_{a r t}}(s)}, \quad T(s)=\frac{N_{T}(s)}{D_{T}(s)} .
$$

With that, the Diophantineequation can be solved and the controller $\mathrm{C}(\mathrm{s})$ can be found as

$$
D_{C}(s) D_{G_{a r t}}(s)+N_{C}(s) N_{G_{a r t}}(s)=D_{T}(s) .
$$

Theexternal gain $k$ and the parameter $\omega_{0}$ in $D_{T}(s)$ provide some flexibility to adjust the behavior (speed, overshoot) of the control system. The so found continuous time controller can be converted to a discrete time controller $C(z)$ and further to a difference equation which can be implemented in a DSP or microcontroller. The transformation from continuous time to discrete time steps is done as follows.

$$
\left.\frac{C(s) 1 \text { tustin }}{s} \stackrel{\text { inv. Z-transform }}{\longrightarrow} \mathrm{C}(\mathrm{z})_{----------} \mathrm{n}\right]
$$

To capture the whole dynamic behavior of the mirror, the sampling frequency (fs $=1.7 \mathrm{kHz}$ ) for the discrete time controller was chosen 10 times larger than the eigenfrequency of the mirror $(f \sim 117 \mathrm{~Hz})$.

\section{EXPERIMENTS AND RESULTS}

The experiments were carried out, using the Texas Instruments TALB1000B MEMS mirror, our proprietary driver electronics and a dsPIC33F digital signal processor from Microchip. The driver electronics include the Digital-to-Analog converters, the power amplifiers and the signal conditioning (noise filtering) of the measurement signals. A block diagram of the whole control loop is shown in Figure 5 . 


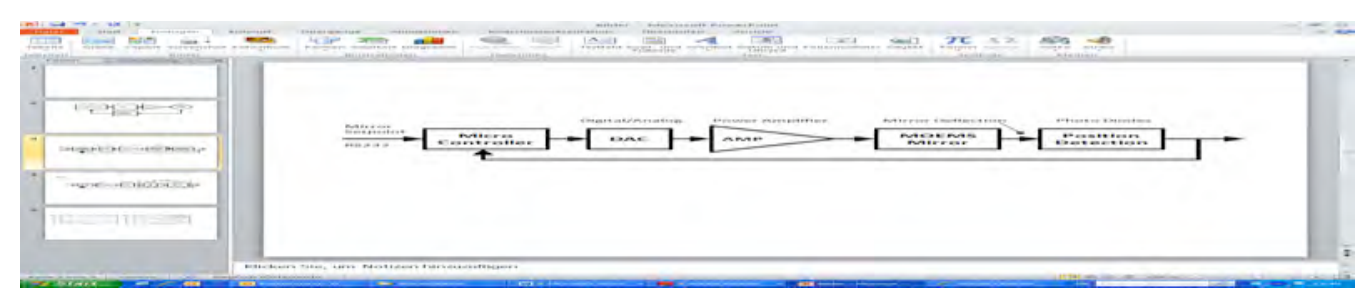

Figure 5:Control loop block diagram

The experimental results show very nice agreement between the simplified mirror model and the real mirror. As a consequence the experimental results for the closed loop step response match the simulation results pretty good. Figure 6 shows the simulated and the experimental step responses for open-loop and various closed-loop strategies. The measurement results are unfiltered and therefore include some noise. In the figure the pole placement controller is named "Algebraic Controller". An open-loop input step results in nearly $100 \%$ overshoot and a settling time of 1.5 seconds. By using the above described closed-loop controller (algebraic or pole placement strategy) the overshoot was reduced to $5 \%$ and the settling timeto 0.05 seconds. This is an improvement by a factor of 30 concerning the positioning speed. Due to the simple control algorithm also less powerful DSPs or microcontrollers can be used for implementing this discrete time controller. The saved computing time could also be used for discrete signal filtering or other additional calculations.
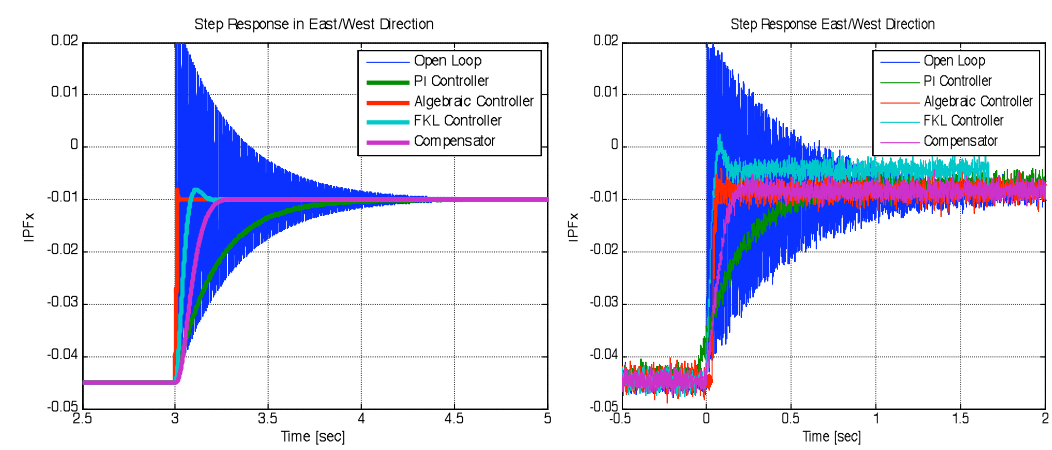

Figure 6 Left: Simulated step responses. Right: Experimental step responses

A good performance concerning step reference inputs is desireable if quasi-static mirrors are used to track arbitrary setpoints. Furthermore, MOEMS mirrors are often operated in a continuous scanning mode. In this case the mirror needs to track periodic signals like sinus or triangular functions. Our control algorithm has also been tested to track periodic triangular reference signals. Without adaptions the controller is able to track triangular signals up to $22.5 \mathrm{~Hz}$. A comparison between simulation and experimental results is shown inFigure 7 . The experimental results have been low pass filtered but still contain some measuerment noise. Due to the fact that the control system is optimized for step reference signals and that it contains only one integrating element,it is clear, that there is a tracking error for triangular signals. This tracking error could be minimized by adaption of the control algorithm. Improvements could be achieved easily by increasing the gain or changing the pole locations of the closed loop system. 


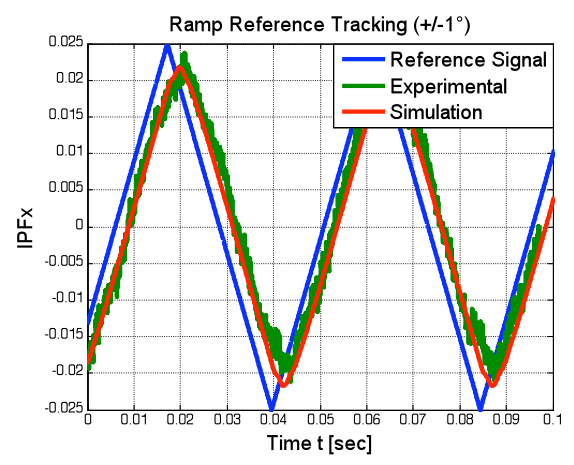

Figure 7: Ramp reference tracking

\section{CONCLUSION \& OUTLOOK}

Discrete-closed loop control of electromagnetically driven MOEMS mirrors was presented is this article. The closed loop control improves the settling time to one tenth of the open loop behavior. Accurate positioning is possible by using a feedback loop. The DSP based controller enables easy and fast tuning and adaption of the controller to the desired behavior and the specifications of different MOEMS mirrors. Setpoint and ramp reference tracking can be realized. It was shown that traditional control algorithms, which are straight forward to implement, can be used to control MOEMS devices.

\section{ACKNOWLEDGEMENTS}

This project was co-financed within the Austrian Kplus/COMET Competence Centre programme. Funding and cooperation with all scientific and industrial partners involved in the 7th framework project "TACO" (grant agreement $n^{\circ} 248623$ ) is gratefully acknowledged.

\section{REFERENCES}

[1] M. Lenzhofer, A. Tortschanoff,Analog driver for synchronized quasi-static MOEMS mirrors, Proc. 22nd Micromechanics and Microsystems Europe Workshop, 2011.

[2] Texas Instruments, Analog Mirror Driver and Estimating Feedback PositionController, Application report, http://www.ti.com/lit/an/dlpa009/dlpa009.pdf, 2009.

[3] Texas Instruments, Dual-Axis Analog MEMS Pointing Mirror, Datasheet, http://www.ti.com/lit/ds/slbs006a/slbs006a.pdf, 2009.

[4] Chi-TsongChen, Analog and Digital Control Systems, Saunders College Publishing, ISBN 0-03094070-2, pp. 367-ff,1993.

[5] S. Kittin, Positionsregelung von Kippspiegeln mit vertikalen Kammantrieben, Diplomathesis, http://publica.fraunhofer.de/dokumente/N-119029.html, 2009

[6] J. Bryzek, E. Abbott, A. Flannery, D. Cagle, J. Maitan, Control Issues for MEMS, Proceedings of the 42nd IEEE Conference on Decision and Control, Maui, Hawaii USA, pp. 3040-3043, December, 2003.

[7] B Borovic, A Q Liu, D Popa, H Cai, F L Lewis, Open-loop versus closed-loop control ofMEMS devices: choices and issues, Journal of Micromechanics and Microengineering, 15,(2005), pp. 1917-1924. 